\title{
p53 and its younger siblings
}

\author{
RA Knight ${ }^{*, 1}$ \\ ${ }^{1}$ Department of Cystic Fibrosis, National Heart and Lung Institute, Imperial \\ College, Emmanuel Kaye Building, Manresa Road, London SW3 6LR \\ * Corresponding author: RA Knight, Department of Cystic Fibrosis, National \\ Heart and Lung Institute, Imperial College, Emmanuel Kaye Building, Manresa \\ Road, London SW3 6LR; Tel: 0171351 8333; Fax: 0171351 8331/8340
}

It happens like this sometimes in families. You get used to being an only child, pampered and studied by almost a generation of scientists, and well on the way to finding your own niche in the biosphere. Just when you're becoming hailed as the guardian of the genome, however, the two strands of DNA that are your parentage reveal that you have two much younger siblings, p63 and p73. Such is the situation with p53. Our current knowledge of this enlarged p53 family is reviewed in this issue ${ }^{1-5}$ As with siblings, so with members of gene families, people first look for likeness. And, indeed, there are some similarities between p53, p63 and p73. They share significant homologies in their DNA binding, transactivation and oligomerisation domains, suggesting that all three function as transcription factors and may transactivate common target genes. There is already evidence that p73, for example, activates the promoters of some genes involved in the cell cycle and apoptosis that are also activated by p53, though quantitative and qualitative differences have been found. Moreover, in the somewhat artificial situation where p73 is overexpressed in p53-deficient cells, apoptosis is induced, suggesting that $\mathrm{p} 73$ at least may be able to substitute for $\mathrm{p} 53$ in the induction of cell death. Not p53, the guardian of the genome, but the p53 family, the guardians of the genome?

However, there is evidence cautioning against this cosy generalisation. Not all forms of DNA damage activate p73, and while p53 mutations in human cancers are common, p63 and p73 mutations are extremely rare. Moreover, the activity of these three proteins depends on their stability/phosphorylation state mediated by co-factors such as MDM-2 and c$\mathrm{Abl}$, as well as some viral proteins. The relative contribution of p53, p63 and p73, and the role of their co-factors, to the induction of cell cycle arrest and apoptosis in response to different stressors therefore remains to be clarified. This is important since, as Arrowsmith points out, we need to understand why in tumours where p53, but not p63 and p73, is mutated, the younger siblings fail in their role as putative substitute guardians of the genome.

These subtle differences in sibling function are accentuated by the additional C-terminal sequences in p63 and p73 which have no homologue in p53. These Cterminal regions contain a sterile alpha motif (SAM) which has been associated in other proteins with regulation of differentiation, and evidence for a developmental role of p63 and p73 is also reviewed here. In addition, and again unlike the single transcript $p 53$, at least six variants of p63 and p73 are generated by alternative splicing involving the C-terminal domain. How this alternative splicing is regulated or whether it is a random event is unknown. Since the C-terminus contains a transactivation motif, it is possible that, as with some members of the CCAAT enhancer binding transcription factors, ${ }^{6}$ differently spliced forms may modulate the transcriptional activity of others. The relationship between this $\mathrm{C}$-terminal and the $\mathrm{N}$-terminal transactivator homologous to p53 is also currently obscure. However, it may be significant that two of the rare p73 mutations have been found in the $\mathrm{C}$-terminal transactivation domain in neuroblastoma, a tumour which can be induced to differentiate in vitro by overexpression of $p 73$.

Homology between sibling genes in their oligomerisation domain implies the potential for the formation of heteromers, a common feature of many other families of transcription factors. However, while there is now good evidence for the homotetramisation of p53, p63 and p73, there is less evidence for heteromer formation and of its physiological significance. Moreover, although SAM motifs have been shown to mediate homo- and hetero-merisation in other proteins, the current evidence suggests that those in the C-terminal regions of p63 and p73 are not involved in this process. Understanding of the molecular interactions between these proteins has important consequences, for example, predicting whether overexpression of p63/p73 can overcome the dominant negative effects of mutant p53 in tumours.

So while we still have a lot to learn before p63 and p73 can be accorded the scientific maturity and respect of their older sibling, it is already clear that they themselves have some distinct functional identities which are different from p53. Understanding p63 and p73 is complicated by their multiply spliced personalities and the interactions between these isoforms. Understanding the relationship between these three siblings and with other non-kin cellular and viral proteins in the regulation of cell growth, differentiation and death is indeed a millenial task.

\section{References}

1. Levrero M, De Laurenzi V, Costanzo A, Gong J, Mellino G and Wang JYJ (1999) Structure, function and regulation of p63 and p73. Cell Death Differ. 6: 11461153

2. Arrowsmith CH (1999) Structure and function in the p53 family. Cell Death Differ. 6: $1169-1173$

3. Shaul Y (1999) c-Abl: activation and nuclear targets. Cell Death Differ. In press

4. Lohrum MAE and Vousden KH (1999) Regulation and activation of p53 and its family members. Cell Death Differ. 6: 1162-1168

5. Ikawa S, Nakagawara A and Ikawa $Y$ (1999) p53 family genes: structural comparison, expression and mutation. Cell Death Differ. 6: 1154-1161

6. Zahnow CA, Younes P, Laucirica R and Rosen JM (1997) Overexpression of C/ Ebp beta-LIP, a naturally occurring, dominant-negative transcription factor, in human breast cancer. J Natl Cancer Inst 89: 1887-1891 